\title{
Uterine histology of the dasyurid marsupial, Antechinus stuartii: relationship with differentiation of the embryo
}

\author{
Y. P. Cruz ${ }^{*}$ and L. Selwood \\ Department of Zoology, La Trobe University, Bundoora, Victoria 3083, Australia
}

\begin{abstract}
Uterine samples from Antechinus stuartii on days 1, 4, 6, 8, 11, 13, 15, 18, 21 and 23 after ovulation were examined histologically. Animals were pregnant, nonpregnant and unmated, or nonpregnant and mated but found to have only unfertilized eggs on autopsy. The histological parameters used were thickness of the myometrium, endometrial stroma, and endometrial epithelium, and density of uterine stromal glands and of lymphocytes at the endometrial basal lamina. Overall, the fluctuation patterns of these parameters were superficially similar between pregnant and nonpregnant animals (mated or unmated). However, statistically significant differences were detected between pregnant and unmated nonpregnant animals in every parameter examined at nearly every time point except day 13 . Comparison of these results with known data on embryonic stages, corpus luteum development and plasma progesterone concentrations revealed that the gravid uterus underwent histological changes co-incident with changes in both progesterone concentration and developmental delay or embryonic arrest. It was concluded that the uterus mediates progesterone-driven changes in embryonic developmental rate. Although determination of number of lymphocytes provided inconclusive evidence of cellular immunity against embryos, the possibility that embryonic signalling to the uterus occurs is discussed.
\end{abstract}

\section{Introduction}

The marsupial reproductive pattern is characterized by a short gestation and a prolonged lactation period. In many known marsupials except macropodids (kangaroos and wallabies), gestation and parturition occur within the luteal phase of the oestrous cycle, which commences with a variably long follicular and ends with a brief post-luteal phase. Neither gestation nor parturition interrupts the oestrous cycle; lactation prevents renewal of the follicular phase. Thus, removal of pouch young from lactating females, and not parturition, results in resumption of the oestrous cycle in polyoestrous species (reviewed in Sharman, 1970; Lee et al., 1982; Tyndale-Biscoe and Renfree, 1987). In monoestrous marsupials, however, removal of pouch young does not provoke resumption of oestrus. In these animals, the follicular phase is not renewed until the next breeding season, as has been shown for Antechinus stuartii (Woolley, 1966).

The oestrous cycle in marsupials is largely indifferent to perturbation by pregnancy. In Didelphis virginiana, a polyoestrous, polyovular species in which ovulation is spontaneous, the uterine anatomical and histological profile of pregnant animals is similar to that of nonpregnant animals until the terminal stages of pregnancy, when implantation occurs (Fleming and Harder, 1981). There appears to be no endocrine recognition of pregnancy in this species; oestradiol:progesterone ratios are about the same on any given day of the oestrous cycle in both

*Present address: Department of Biology, Oberlin College, Oberlin, OH 44704, USA.

Received 29 December 1992 pregnant and nonpregnant animals (Harder and Fleming, 1981). In polyoestrous dasyurid marsupials, the pattern of changes in body weight, plasma progesterone and uterine histology during the luteal phase which follows the spontaneous ovulation are similar in pregnant and nonpregnant animals (reviewed in Tyndale-Biscoe and Renfree, 1987).

Physiological and anatomical differences have been observed, however, between the gravid and non-gravid uteri of pregnant, monovular, polyoestrous marsupials. In Trichosurus vulpecula, for example, the mitotic rate is higher in the endometrium of the gravid uterus (von der Borch, 1963), suggesting that the Graafian follicle may have a local hormonal effect on the ipsilateral uterus (McDonald and Waring, 1982; Young and McDonald, 1982; Curlewis and Stone, 1986). The gravid uterus weighs more in this polyoestrous species, a condition attributed to increased progesterone concentrations in the uterine circulation (Curlewis et al., 1985). High progesterone may in turn result from increased synthesis of progesterone receptors which, in Setonix brachyurus (Owen et al., 1982) and Macropus eugenii (Renfree, 1973), appears to be induced by oestrogen (Flint and Renfree, 1982; Harder et al., 1984; Shaw and Renfree, 1984).

The dasyurid marsupial, Antechinus stuartii, is monoestrous (Marlow, 1961; Woolley, 1966), polyovular and polytocous (Selwood, 1980). Ovulation is spontaneous (Woolley, 1966); the 27-day gestation period is relatively long (Selwood, 1980). The rate of embryonic development appears to parallel that of corpus luteum formation and is positively correlated with concentrations of progesterone in plasma (Hinds and Selwood, 1990). Woolley (1966) suggested that the rate of corpus luteum 
Table 1. Number of female Antechinus stuartii used as source of uterine samples in this study

\begin{tabular}{|c|c|c|c|c|c|c|c|c|c|c|c|}
\hline \multirow[b]{2}{*}{ Status of animal } & \multicolumn{11}{|c|}{ Days after ovulation } \\
\hline & 1 & 4 & 6 & 8 & 11 & 13 & 15 & 18 & 21 & 23 & Total \\
\hline Pregnant $(\mathrm{P})$ & 2 & 1 & 1 & 2 & 1 & 1 & 2 & 5 & 4 & 2 & 21 \\
\hline $\begin{array}{l}\text { Not pregnant/ } \\
\text { mated (NPM) }\end{array}$ & 1 & 0 & 0 & 0 & 1 & $\mathrm{I}$ & $\mathrm{I}$ & 1 & 1 & 1 & 7 \\
\hline $\begin{array}{l}\text { Not pregnant/ } \\
\text { unmated (NPU) }\end{array}$ & 0 & 1 & 1 & 1 & 0 & 0 & 0 & 0 & 0 & 0 & 3 \\
\hline Total & 3 & 2 & 2 & 3 & 2 & 2 & 3 & 6 & 5 & 3 & 31 \\
\hline
\end{tabular}

development controls the rate of embryonic development, which in A. stuartii is arrested in vivo and in vitro at the fourcell stage and the stage of the early expanding unilaminar blastocyst (Selwood 1981; Selwood and Young, 1983). Because progesterone-supplemented culture medium does not terminate this developmental arrest (Selwood, 1987), Hinds and Selwood (1990) suggested that, if progesterone plays a role in regulating embryonic development, its action must be mediated via the uterus. In this report, we evaluate the differences between the uterine myometrium and endometrium of pregnant and nonpregnant $A$. stuartii during the luteal phase at known stages of gestation. We then correlate these differences with the day after ovulation, the developmental stages of embryos, and with previously determined concentrations of progesterone in plasma during pregnancy.

\section{Materials and Methods}

Uteri used in this study were from animals maintained in laboratory colonies at La Trobe University during 1975, 1976, 1978, 1981-1984, 1987 and 1988 under Ministry of Conservation and Natural Resources permit numbers $75 / 237,76 / 91,78 / 428$, $81 / 110,82 / 32,82 / 180,84 / 31,85 / 107$ and $87 / 56$ used for other studies. As such colonies are no longer maintained, the material used was restricted to that already collected.

Uterine samples were obtained from 31 animals determined by urine sampling to be at specific stages after ovulation, following the method described by Selwood (1980). Of these animals, 21 were pregnant $(\mathrm{P})$, seven nonpregnant (unmated) (NPU), and three nonpregnant (mated but found upon dissection to have unfertilized eggs) (NPM) (Table 1). Animals on days $1,4,6,8,11,13,15,18,21$ and 23 after ovulation were individually anaesthetized with sodium amytal (Lilly, Indianapolis), at $0.08-0.10 \mathrm{mg} \mathrm{g}^{-1}$ body weight (Woolley, 1966). The uteri were excised, rinsed in phosphate-buffered saline and later dissected in serum-supplemented culture medium, into which any embryos present were collected, examined (Selwood, 1980) and used in other studies. The number of corpora lutea in each ovary was determined. This procedure provided additional confirmation that ovulation had occurred and, in the case of pregnant animals, that each uterus was gravid.
Uteri were fixed in Bouin's fixative (Humason, 1967) for $24 \mathrm{~h}$, dehydrated in alcohol and embedded in paraffin. Transverse serial sections at $8 \mu \mathrm{m}$ were taken of the uterus from the oviduct (anterior) end to a point midway between the anterior and posterior ends of the uterus, where it was widest. Sections were stained in haematoxylin and eosin (BDH Australia, Kilsyth, Victoria) and mounted in Depex (BDH Australia, Kilsyth, Victoria). From each set of slides representing the widest part of a given uterus, a section was chosen randomly. This and two other sections approximately 100 and $200 \mu \mathrm{m}$ away from the first were examined under brightfield microscopy (Wild M40 inverted microscope, Wild Australia, Prahran, Victoria). Representative sections were photographed using Kodak TMX film, 100 ASA.

The tissues in each uterine section were examined following a uniform procedure for each. Myometrium thickness was measured along a transect drawn perpendicularly between the roughly parallel distal and proximal surfaces of the myometrium. Endometrial stroma thickness was measured in a similar manner, as was the thickness of the endometrial epithelium, which lines the uterine lumen. The density of endometrial glands was estimated by counting the number of sectioned glands encountered along a transect drawn perpendicularly relative to the surfaces of the endometrial stroma. Three sets of these measurements were obtained from each animal. Lymphocytes were counted in a grid superimposed on the sectioned tissue, with one edge of the grid positioned in perfect register with the basal lamina of the endometrial epithelium. A total of ten randomly selected grids, distributed among the three sections evaluated, were counted for every animal.

The numbers of animals examined on specified days after ovulation are shown (Table 1). Effects of reproductive status on uterine parameters were compared by a $t$ test. Because the material was examined retrospectively after colonies were disbanded, it was not possible to increase the number of animals examined.

\section{Results}

The histological differences detected among uteri from P, NPU, and NPM animals are summarized (Fig. 1). The reproductive 


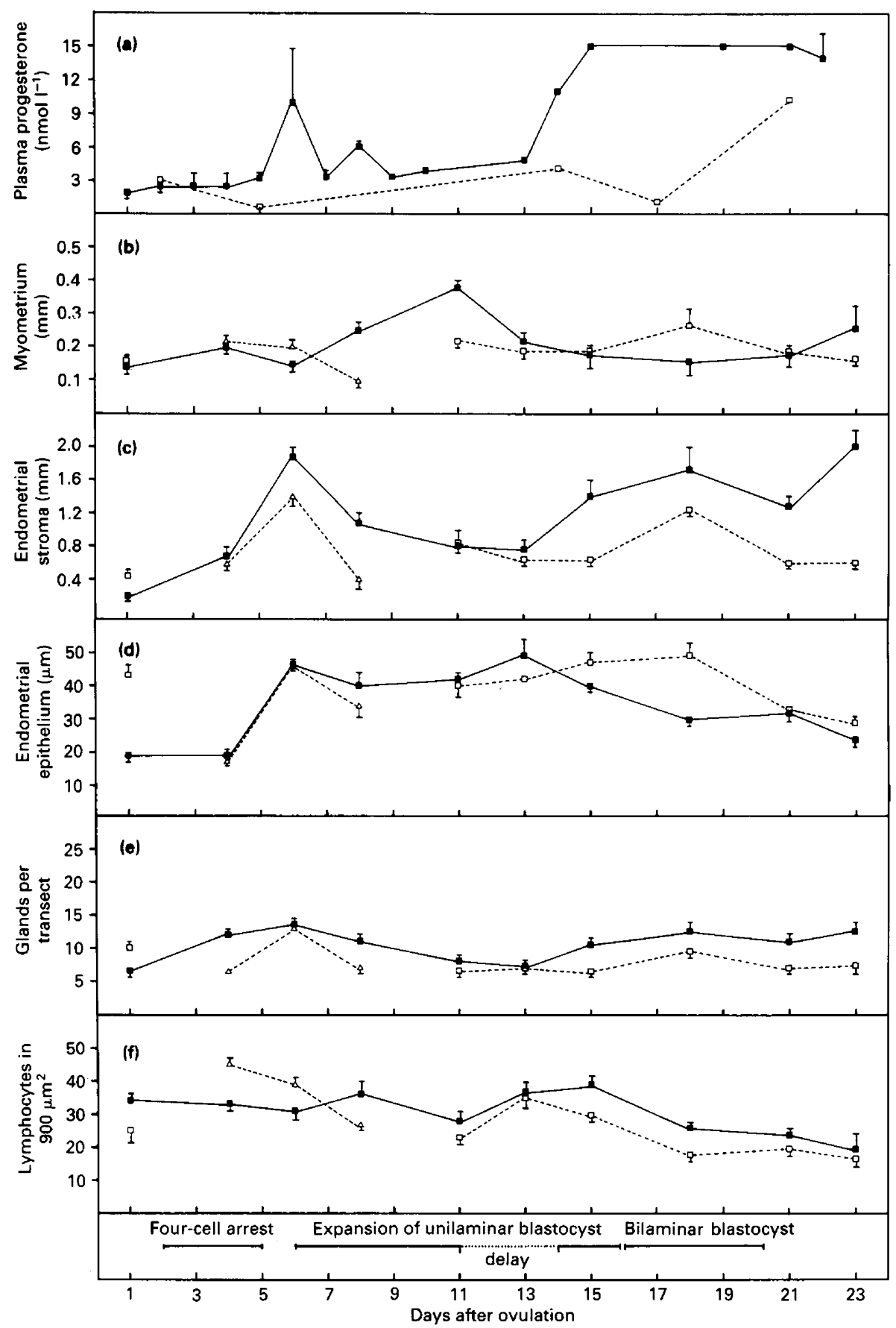

Fig. 1. Uterine histological profile and plasma progesterone levels of $(\boldsymbol{\square})$ pregnant, $(\Delta)$ nonpregnant and mated, and ( $\square$ ) nonpregnant and unmated Antechinus stuartii on various days after ovulation. (a) Plasma progesterone concentrations (data from Hinds and Selwood, 1990); (b) thickness of myometrium; (c) thickness of endometrial stroma; (d) thickness of endometrial epithelium; (e) number of stromal glands per transect; (f) density of lymphocytes at basal lamina of endometrial epithelium. Means are presented \pm SEM. Relevant stages of embryonic development are indicated.

states of the animals (days after ovulation) are shown alongside the developmental stages of embryos retrieved from them. Plasma progesterone concentrations from animals of identical reproductive state (days after ovulation) are from Hinds and Selwood (1990).

\section{Myometrium}

Myometrial thickness fluctuated in P and NPU animals, that in the former rising by day 8 to a peak on day 11 . The myometrium of NPU animals was thickest on day 18 and was 

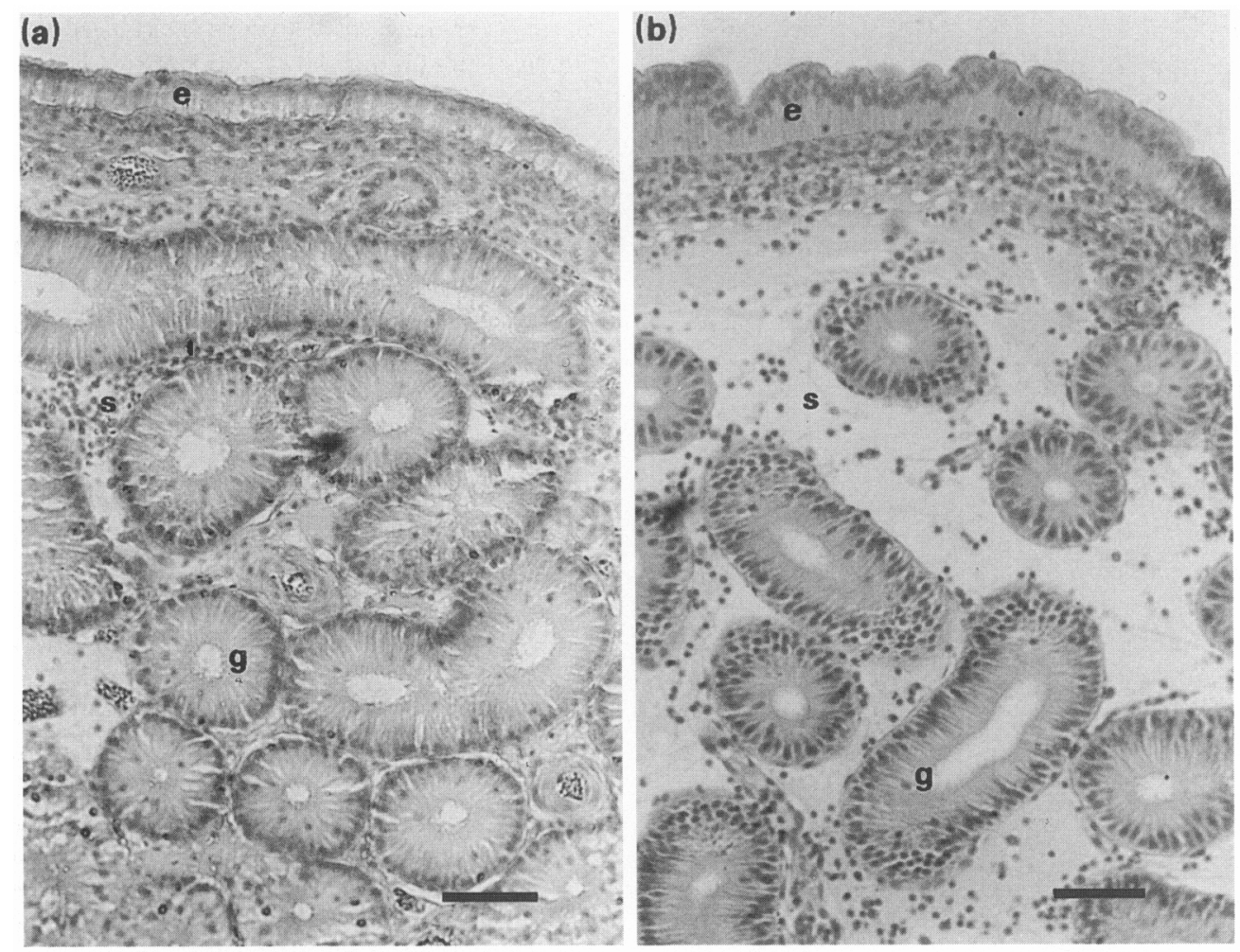

Fig. 2. Transverse section of uterine wall from (a) pregnant and (b) nonpregnant Antechinus stuartii 18 days after ovulation showing (a) $\mathbf{e}$, thin endometrial epithelium; $\mathbf{g}$, stromal glands with highly polarized secretory cells; and s, endometrial stroma densely packed with stromal glands and (b) e, thick endometrial epithelium; $\mathbf{g}$, stromal glands with moderately polarized secretory epithelium; and s, oedematous endometrial stroma loosely packed with stromal glands. Scale bars $=50 \mu \mathrm{m}$.

significantly thicker $(P<0.05)$ than that of $P$ animals. No obvious histological differences were observed among the myometrial vascular systems of uteri from P, NPU and NPM animals.

\section{Endometrial stroma}

Great fluctuations in thickness characterized the endometrial stroma in the three groups of animals examined. The stroma of $P$ animals exhibited more than a sevenfold increase in thickness, and was thinnest on day 1 and thickest on days 6,18 and 23. The peaks of thickness were mirrored by the stroma of both NPU and NPM animals, although the stroma of P animals was consistently and significantly $(P<0.05, P<0.01$, or $P<0.001)$ thicker at every peak.

\section{Endometriat epithelium}

The endometrial epithelium was low columnar and pseudostratified in all sections examined. In NPU animals, it was as thick as, or thicker than, that in $\mathrm{P}$ animals at any time after ovulation for which comparisons were available. Epithelial thinning in gravid uteri was significant $(P<0.05)$ on day 15 and very highly so $(P<0.001)$ on days 1 and 18 , and appeared to be due to loss of cytoplasmic material on the lumen side of the epithelial surface, which exhibited a ragged outline (Fig. 2a).
This contrasted sharply with the finely villous surface of the endometrial epithelium characteristic of the non-gravid uterus (Fig. 2b).

\section{Endometrial glands}

Gland density was significantly greater $(P<0.05$ on day 11 , $P<0.01$ on days 18 and $23, P<0.001$ on days $4,8,15$ and 21) in P than in NPU or NPM animals on every day of gestation examined except on days 1,6 and 13. Mitotic figures were observed in the gland epithelium from both gravid and nongravid uteri at the rate of about one per transverse section (containing from 50 to 300 cells) until day 6 , and at frequencies less than that thereafter. On days 16 and 18, haematoxylin-stained, translucent patches were evident in the lumen of endometrial glands from $\mathrm{P}$ animals, suggesting the presence of proteinaceous fluid.

\section{Lymphocyte density at endometrial basal lamina}

Lymphocytes were clustered in a dense mat in the uterine stroma adjacent to the basal lamina of the endometrial epithelium (Fig. 2). Lymphocyte counts were roughly equal in $P$ and NPU animals, although significantly greater in the former on days $I(P<0.05)$ and $15(P<0.01)$. After day 15 , lymphocyte counts declined in both $P$ and NPU animals, more abruptly 
in the latter. Lymphocyte density in NPM animals decreased sharply from days 4 to 8 , exhibiting a trend completely the reverse of that in $\mathrm{P}$ animals (Fig. I).

\section{Discussion}

The gravid uterus of $A$. stuartii was shown to be histologically distinguishable from its non-gravid counterpart. Although uterine histological profiles from P, NPU and NPM animals were superficially similar, careful inspection revealed that statistically significant differences were detectable at all time points examined except day 13. This instance of minimal (or no) uterine histological differences immediately precedes the period of sustained increase in plasma progesterone in pregnant animals which commences on day 13 . This period coincides with the termination of slow or arrested development typical of $A$. stuartii embryos at the expanding, unilaminar blastocyst stage (Hinds and Selwood, 1990). Another time point during which histological differences between gravid and non-gravid uteri are minimal is day 4. Again, this time point immediately precedes, in pregnant animals, a modest but distinctive rise in concentrations of progesterone in plasma (Hinds and Selwood, 1990) and coincides with the termination of arrested development in four-cell A. stuartii embryos (Selwood, 1980). It is clear, therefore, that increased concentrations of plasma progesterone correlate strongly not only with resumption of embryonic development, but also with changes in the histological profile of the gravid uterus.

These results are inconsistent with results for Didelphis virginiana, the only other marsupial species in which the uterine histology concentrations of progesterone in plasma and corpus luteum development of pregnant and nonpregnant animals have been compared at matched time-points. In $D$. virginiana, gravid uteri differ from non-gravid uteri in one aspect and in one instance only. On day 11 (2 days before parturition), the uterine stroma is significantly thinner in pregnant animals (Fleming and Harder, 1981). An equivalent stage was not sampled in $A$. stuartii but the uterine stroma in $A$. stuartii is considerably thicker $(P<0.01)$ in pregnant animals on day 23 , the day of implantation, 3-4 days before parturition.

There is an important point of similarity between $D$. virginiana and $A$. stuartii, however. In both species, concentrations of progesterone in plasma clearly reflect the developmental state of the corpus luteum, in which secretory activity reaches a peak value towards the end of pregnancy (Hartman, 1928; Harder and Fleming, 1981; Hinds and Selwood, 1990). This three-point correlation among corpus luteum, progesterone and embryonic development also occurs in Dasyurus viverrinus (Sandes, 1903; Hinds, 1989). In addition, concentrations of progesterone in plasma have been shown to correlate positively with corpus luteum development in Trichosurus vulpecula (Thorburn et al., 1971) and endometrial weight in D. virginiana (Renfree, 1975).

The uterus is the link between progesterone production and embryonic development. The gravid uterus of $A$. stuartii, however, presents a histological profile quite different from that of non-gravid uteri, suggesting two possibilities: (i) the presence of embryos alters the uterine milieu and, ultimately, its histological profile or (ii) the progesterone rise between days 13 and 15 in pregnant animals provokes greater changes in the histological profile of the uterus. An embryonic signal would be necessary in either case. In the latter, it would have to be directed at the corpus luteum. The nature of the signal and precisely how it would be transmitted are unclear.

It is clear, however, that embryonic signalling occurs during pregnancy. In eutherian animals, in which implantation typically occurs relatively early in gestation, chorionic gonadotropin production by embryonic trophoblast cells signals the corpus luteum to remain actively secreting progesterone (Dorrington, 1979). This does not occur in marsupials (Tyndale-Biscoe and Renfree, 1987) perhaps because implantation is late or of short duration. The observations that corpus luteum development is the same in pregnant and nonpregnant $D$. virginiana (Fleming and Harder, 1983) and T. vulpecula (Pilton and Sharman, 1962) suggest strongly that embryonic signalling, if it occurs, bypasses the corpus luteum.

The presence of acellular investments around the marsupial embryo possibly presents a barrier to signal transmission. Although these investments (zona pellucida, mucoid layer, shell) are permeable to diverse substances, progressively become attenuated, and eventually disappear during pregnancy, they may restrict communication between embryo and uterus to the extent that an immunoprotective role is ascribed to them (reviewed in Tyndale-Biscoe and Renfree, 1987). The comparison of lymphocyte density reported in this study appears to confirm this view, albeit inconsistently, suggesting that a cellular immune response to pregnancy is not manifested in the endometrial basal lamina. We note, however, that lymphocyte counts from pregnant animals are high on day 15, when the primitive endoderm forms in A. stuartii embryos (Selwood, 1980) and novel proteins would be synthesized. Perhaps the earliest immune response to pregnancy in marsupials is humoral, or the cellular immune response is manifested in the peripheral circulation (O'Neill, 1985; O'Neill et al., 1985) or in adjacent lymphatics (Clarke, 1984).

In conclusion, gravid and non-gravid uteri exhibit significant histological differences that correlate with embryonic developmental rate, concentrations of progesterone in plasma and corpus luteum formation in $A$, stuartii.

The authors are grateful to I. Douglas and V. Renshaw for technical assistance. Y. P. Cruz was funded by a Research Status grant from Oberlin College and L. Selwood by a grant from the Australian Research Council.

\section{References}

Clarke AG (1984) Immunological studies on pregnancy in the mouse. In Immunological Aspects of Reproduction in Mammals pp 153-182 Ed. DB Crichton. Butterworths, London

Curlewis JD and Stone GM (1986) Effects of oestradiol, the oestrous cycle and pregnancy on weight, metabolism and cytosol receptors in the uterus of the brush-tail possum (Trichosurus vulpecula) Journal of Endocrinology 108 201-210

Curlewis JD, Axelson M and Stone GM (1985) Identification of the major steroids in ovarian and adrenal venous plasma of the brush-tail possum (Trichosurus vulpecula) and changes in the peripheral plasma levels of oestradiol and progesterone during the reproductive cycle Journal of Endocrinology 105 53-62

Dorrington JH (1979) Pituitary and placental hormones. In Reproduction in Mammals, Vol. 7. Mechanisms of Hormone Action pp 53-80 Eds CR Austin and RV Short. Cambridge University Press, Cambridge 
Fleming MW and Harder JD (1981) Uterine histology and reproductive cycles in pregnant and non-pregnant opossums, Didelphis virginiana Journal of Reproduction and Fertility $6021-24$

Fleming MW and Harder JD (1983) Luteal and follicular populations in the ovary of the opossum (Didelphis virginiana) after ovulation Joumal of Reproduction and Fertility $6729-34$

Flint APF and Renfree MB (1982) Oestradiol-17 $\beta$ in the circulation during seasonal inactivation of the diapausing blastocyst in a wild population of tammar wallabies, Macropus eugenii Joumal of Endocrinology 95 293-300

Harder JD and Fleming MW (1981) Estradiol and progesterone profiles indicate a lack of endocrine recognition of pregnancy in the opossum Science $\mathbf{2 1 2}$ 1400-1402

Harder JD, Hinds LA, Horn CA and Tyndale-Biscoe CH (1984) Oestradiol in the follicular fluid and in utero-ovarian venous and peripheral plasma during parturition and post-partum oestrus in the tammar, Macropus eugenii Joumal of Reproduction and Fertility 72 551-558

Hartman CG (1928) The breeding season of the opossum (Didelphis virginiana) and the rate of intrauterine and postnatal development Journal of Morphology and Physiology 46 143-215

Hinds LA (1989) Plasma progesterone through pregnancy and the oestrous cycle in the eastern quoll, Dasyurus viverrinus General and Comparative Endocrinology 75 110-117

Hinds LA and Selwood L (1990) Plasma progesterone concentrations during pregnancy in the dasyurid marsupial, Antechinus stuartii: relationship with differentiation of the embryo Reproduction, Fertility and Development $261-70$

Humason GL (1967) Animal Tissue Techniques (2nd edn) p 14. WH Freeman \& Company, San Francisco

Lee AK, Woolley P and Braithwaite RW (1982) Life history strategies of dasyurid marsupials. In Carnivorous Marsupials Vol. 1, pp 1-11 Ed. M Archer. Royal Zoological Society of New South Wales, Sydney

McDonald IR and Waring H (1982) Hormones of marsupials and monotremes. In Hormones and Evolution Vol. 2, pp 873-924 Ed. EJW Barrington. Academic Press, London

Marlow BJ (1961) Reproductive behavior of the marsupial mouse, Antechinus flavipes (Waterhouse) (Marsupialia) and the development of the pouch young Australian Joumal of Zoology 9 203-218

O'Neill C (1985) Thrombocytopenia is an initial maternal response to fertilization in mice Joumal of Reproduction and Fertility 73 559-566

O'Neill C, Gidley-Baird AA, Pike IL, Porter RN, Sinosich MJ and Saunders DM (1985) Maternal blood platelet physiology and luteal-phase endocrinology as a means of monitoring pre- and post-implantation embryo viability following in vitro fertilization Journal of In Vitro Fertilization and Embryo Transfer 2 87-93

Owen FJ, Cake MH and Bradshaw SD (1982) Characterization and properties of a progesterone receptor in the uterus of the quokka (Setonix brachyurus) Journal of Endocrinology 93 17-24

Pilton PE and Sharman GB (1962) Reproduction in the marsupial Trichosurus vulpecula Journal of Endocrinology 25 119-136

Renfree MB (1973) Proteins in the uterine secretions of the marsupial Macropus eugenii Developmental Biology 32 41-49

Renfree MB (1975) Uterine proteins in the marsupial, Didelphis marsupialis virginiana, during gestation Joumal of Reproduction and Fertility 42 163-166

Sandes FP (1903) The corpus luteum of Dasyurus viverrinus with observations on the growth and atrophy of the Graafian follicle. Proceedings of the Linnean Society of New South Wales 28 364-405

Selwood L (1980) A timetable of embryonic development of the dasyurid marsupial Antechinus stuartii (Macleay) Australian Journal of Zoology 28 $649-668$

Selwood L (1981) Delayed embryonic development in the dasyurid marsupial, Antechinus stuartii Journal of Reproduction and Fertility Supplement 29 79-82

Selwood L (1987) Embryonic development in culture of two dasyurid marsupials, Sminthopsis crassicaudata (Gould) and Sminthopsis macroura (Spencer) during cleavage and blastocyst formation Gamete Research 16 355-370

Selwood L and Young GJ (1983) Cleavage in vivo and in culture in the dasyurid marsupial Antechinus stuartii (Macleay) Journal of Morphology 176 43-60

Sharman GB (1970) Reproductive physiology of marsupials Science 167 1221-1228

Shaw G and Renfree MB (1984) Concentrations of oestradiol-17 $\beta$ in plasma and corpora lutea throughout pregnancy in the tammar, Macropus engenii Journal of Reproduction and Fertility $\mathbf{7 2}$ 29-37

Thorburn GD, Cox RI and Shorey CD (1971) Ovarian steroid secretion rates in the marsupial Trichosurus vulpecula Journal of Reproduction and Fertility $\mathbf{2 4} 139$

Tyndale-Biscoe H and Renfree MB (1987) Reproductive Physiology of Marsupials. Cambridge University Press, Cambridge

von der Borch SM (1963) Unilateral hormone effect in the marsupial Trichosurus vulpecula Journal of Reproduction and Fertility 5 447-449

Woolley P (1966) Reproduction in Antechinus spp. and other dasyurid marsupials Symposium of the Zoological Society of London 15 281-294

Young CE and McDonald IR (1982) Oestrogen receptors in the genital tract of the Australian marsupial Trichosurus vulpecula General and Comparative Endocrinology 46 417-427 